Journal of the Egyptian Society of Parasitology, Vol.43, No.3, December 2013 J. Egypt. Soc. Parasitol., 43(3), 2013: 747 - 754

\title{
FINGERPRINTING AND GENETIC RELATIONSHIP OF TRICHOMONAS VAGINALIS, TRICHOMONAS MURIS AND TRICHOMONAS GALLINAE By
}

MAGDA M. EL-NAZER, NADA A. EL NADI, AMAL M. AHMED AND AMAL A. ABDEL MAWGOOD

Department of Medical Parasitology, Faculty of Medicine. Sohag University, Sohag, Egypt, e-mail correspondence: magdaelnazer@gmail.com

\begin{abstract}
Because of the common pathology, protein expression and drug resistance mechanisms-though in different hosts- of the luminal trichomonads Trichomonas vaginalis, T. gallinae and T. muris, doubts were raised concerning their phylogenetic relationship. The random amplified polymorohic (RAPD) technique for genetic DNA polymorphism studying was used to genetically characterize and assess their interspecies polymorphism. Results showed a high statistical support for the close relationship between the tested parasites and proved a tight association between Tritrichomonas muris and T. gallinae as well as a high genetic similarity between T. muris and T. vaginalis.
\end{abstract}

Key words: phylogeny, RAPD, Trichomonas vaginalis, T. gallinae, T. muris.

\section{Introduction}

Trichomonads are flagellated amitochondrial anaerobic protists, branched very early in the eukaryotic phylogeny. Until 2009; relatively little effort has been invested into molecular studies of the trichomonad diversity (TorresMachorro et al, 2009). Molecular characterization of intestinal protists is necessary to design primers and probes as this is the basic material for current and future improved diagnostic PCRs for either detecting all genetic variants or specifically differentiating among such variants. The advancements were also made in phylogenetic systematics, mapping the transition of traits into a comparative and testable frame-work through the publication and use of evolutionary trees (Wiley and Lieberman,
2011). The random amplified polymorphic DNA (RAPD) technique represents an efficient tool for the study of genetic polymorphism of DNA (Jamali et al, 2005), it can detect polymorphisms in any kind of sequences (Perez et al, 1998).

This method also samples the genome more randomly than conventional methods such as allozyme and RFLP (Lynch and Milligan, 1994). Phylogenetics location of trichomonads remained unclear until 2009 (TorresMachorro et al, 2009).

This work aims to explore the genetic DNA polymorphism and interspecies relationship between 3 trichomonads, namely $T$. vaginalis, $T$. gallinae, $T$, muris of rats and mice. 


\section{Materials and Methods}

In this study, rodent trichomonads (16 isolates from rats and 20 from mice), an isolate of $T$. vaginalis and an isolate of $T$. gallinae were used.

Sample collection and preparation: 1$T$. vaginalis isolates: vaginal washes and aspirations were collected (Barretto et al, 1957). 2- T. gallinae: 2 crop swabs from each suspected pigeon (3-4 weeks old) were taken by sterile cotton swabs (Stabler, 1951). 3- T. muris, stool samples from rats and mice were collected in $3 \mathrm{ml}$ sterile isotonic saline (Schmidt and Roberts, 1996). All samples were divided in two sterile screwcapped centrifuge tubes one for culture inoculation and the other for wet mount examination.

Culture preparation and inoculation: All isolates (-ve and +ve wet mounts) were inoculated in dehydrated culture medium for Trichomonas (CM0161Oxoid Ltd-UK). Culture was prepared according to manufacturer pamphlet.

DNA Extraction: DNA of all (38) isolates were extracted using PrepMan Ultra solution (Applied BiosystemsLife Technologies) according to manufacturer's manual, followed by Nucleic acid precipitation.

RAPD PCR: Six different random primers (Invitrogen-Life Technologies) based on Valadkhani et al. (2011) were used for RAPD Analysis; OPD1 (ACC GCGAAGG), OPD2 (GGACCCAAC C), OPD3 (GTCGCCGTCA), OPD8 (TCCTCACCGACC), Tv2 (TCGGCC GCTATC), Tv6 (GGGACCTACTGC). They were found suitable for analyzing these isolates.
DNA amplification was prepared and performed. at final volume of $25 \mu \mathrm{l}$ containing: $1 \mu \mathrm{l}$ of each primer (InvitrogenLife Technologies) with concentration of 50 pmole $/ 25 \mu 1,2.5 \mu \mathrm{l}$ of template DNA with concentration of $100 \mathrm{ng} / 25$ $\mathrm{ul}, 4 \mu \mathrm{l}$ of nuclease free water (Ambion) and 12.5ul of the Taq PCR Master Mix Kit (cat. nos. 201443 \& 201445), contains all components of PCR reaction except primers and template DNA (2 unit/ $\mu$, Qiagen). Negative controls for each primers used contained all components except template DNA. PCR products were analyzed by electrophoresis in $1.5 \%$ agarose gel (Invitrogen) in TBE buffer and DNA stain $(3 \mu \mathrm{l} / 100$ $\mathrm{ml}$ ), gels were then stained with Ethidium bromide $(0.5 \mu \mathrm{g} / \mathrm{ml})$ visualized and photographed. The banding pattern size of each isolates was scored (bp) in compare with size marker in $100 \mathrm{bp}$ DNA ladder (Invitrogen, UK).

Genetic polymorphism analysis: After Vanacova et al. (1997), genetic distances between DNA samples were computed from Nei's coefficients of similarity. Dendrogram based on Ward method was built using SPSS 11.0 program (Jamali et al, 2005).

\section{Results}

RAPD patterns obtained with primers used (Figs. 1, 2, 3, 4, 5). The isolates with similar banding pattern were considered as a single type. Visual comparison of the band profiles on the agarose gels for each of OPD primer sets indicated moderate reproducibility of results. Data were incorporated from 24-41 bands of sizes ranged 200-450 base pair for the OPD primer sets. 
A little indication of interspecies divergence between all isolates is provided by considering the proportion of bands shared between isolates, which is described as the percentage similarity based on Nei's coefficient; with percentage similarity amongst isolates of $>93 \%$ for OPD3 and $>85 \%$ similarity for OPD8. For OPD1, only four isolates (Tmm11, Tmm12, Tmm14 \& Tmm17) had $>90 \%$ similarity, but, the remaining 36 isolates $>37 \%$ similarity. T. gallinae isolate showed $57 \%$ similarity with the tested isolates, for OPD3 and OPD8 primers and with $77 \%$ overall similarity amongst the RAPD band patterns. $T$. vaginalis isolate showed less amount of similarity; $53 \%$ with the isolates tested, and this was consistent for OPD1, OPD3, OPD8 primers sets and with $87 \%$ overall similarity among the RAPD band patterns.

Phylogenetic tree analysis results: According to the phylogenic tree (based on Ward method); the isolates fell into two major groups, the upper branch consisted of 32 isolates, 17 of them were mice Trichomonas muris, 14 were rat $T$. muris and the $T$. gallinae isolate. The lower branch of the tree, consisted of 6 isolates, those were more related to the isolate of Trichomonas vaginalis (Fig. 6).

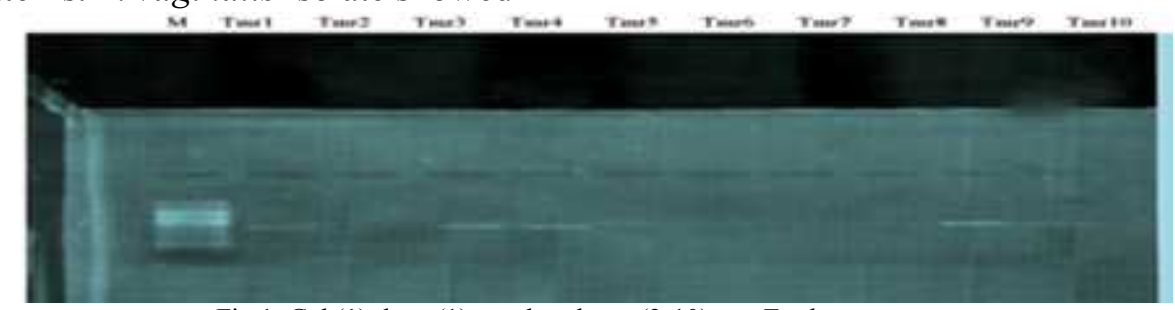

Fig 1: Gel (1): lane (1): marker, lanes (2-10): rat Trichomonas muris

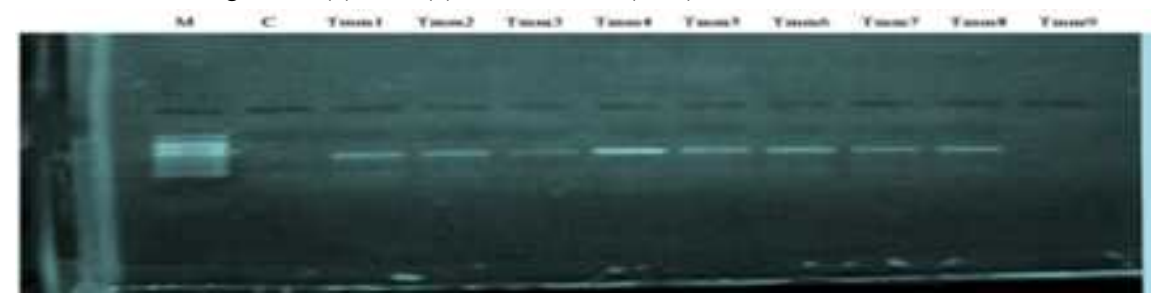

Fig 2: Gel (2):lane (1): marker, lanes (2) : -ve control, lanes (3-10): mice Trichomonas muris.
$\rightarrow$
Tneto twasis
Tramiz $\operatorname{Tamis} 13$
remit Tramis rimels
Tami7 Trmis

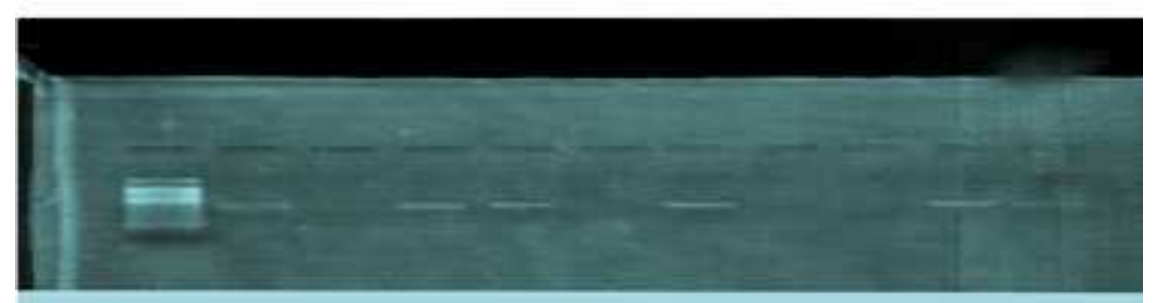

Fig 3: Gel (3): lane (1): marker, lanes (2-11): mice Trichomonas muris 


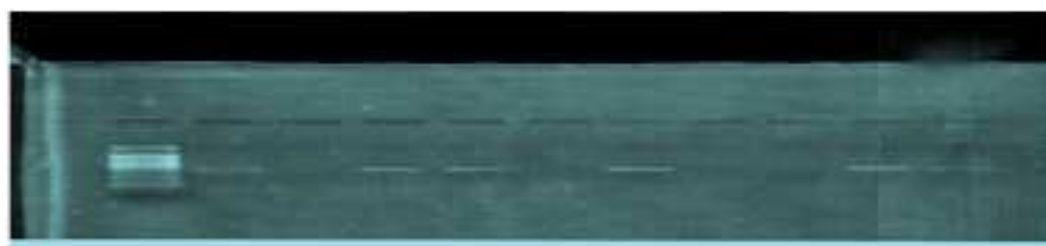

Fig 4: Gel (4): lane(1): marker, lane (2): -ve control, lane (3): T. vaginalis, lanes (4-8) rat T. muris, lanes (9-10): mice T.muris lane (11): T. gallinae .

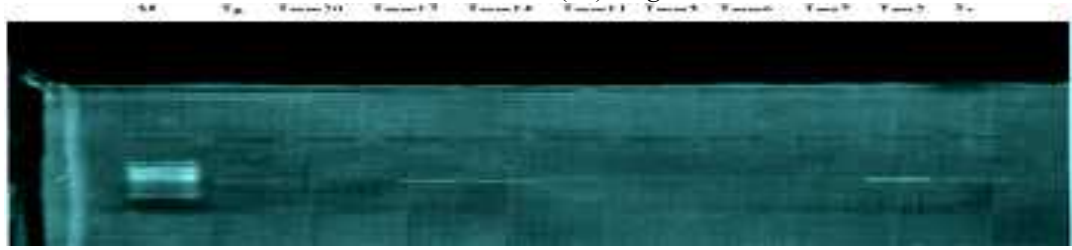

Fig 5: Gel (5): lane (1): marker, lane (2):T. gallinae, lanes (3-8):mice T. muris, lanes (9-10 ): rat T.muris, lane (11), T. vaginalis; by OPD1, OPD3 and TV6 primers.

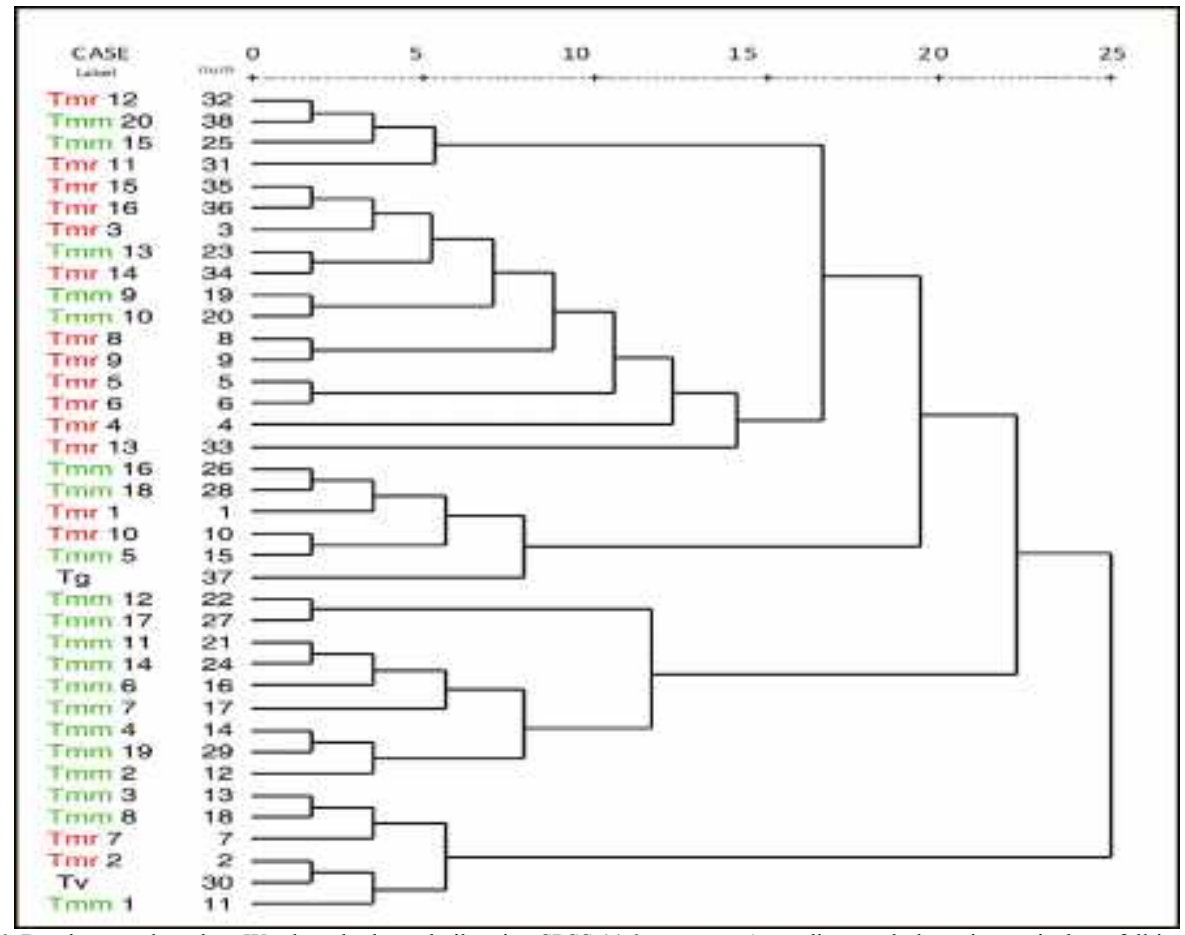

Fig 6: Dendrogram based on Ward method was built using SPSS 11.0 program. According to phylogenic tree isolates fell into two major groups upper branch consisted of 32 isolates including $T$. gallinae isolate. Lower branch of tree, consists of 6 isolates, more related to $T$. vaginalis isolate.

\section{Discussion}

Members of Trichomonas spp. are parasitic protozoa that proliferate on mucous membranes of urogenital, gastro-intestinal or nasal tracts. Several studies identified either novel tricho- 
monad species or trichomonads in an abnormal host or tissues (Mostegl et al, 2012). The present study revealed tight genetic relationships between $T$. gallinae and Tritrichomonas muris, thus supporting the suggestion of cross infection among different species of intestinal trichomonads.

Frey and Muller (2012) reported that previous studies on morphological, physiological and molecular levels have raised doubts as to the phylogenetic relationship among some Tritrichomonas species, particularly in relation to $T$. foetus (of cattle), Tritrichomonas suis (of swine), and Tritrichomonas mobilensis (of primates). With the advent of molecular genetic tools, it has become clear that these three tritrichomonad species are closely related or may even represent the same species. Those doubts as to the phylogenetic relationships among some Tritrichomonas species were raised by the present work which obviated an overall similarity of $>93 \%$ among RAPD bands of $T$. muris, $T$. vaginalis and $T$. gallinae. Also, this similarity percentage raised the doubt of phylogenetic relationships amongst whole trichomonads.

Multiple approaches to typing Trichomonas isolates have been described; antigenic characterization, isozyme analysis, repetitive sequence hybridization, ribosomal gene and intergenic region sequence polymorphisms, pulsedfield gel electrophoresis, random amplified polymorphic DNA analysis, and restriction fragment length polymorphism (RFLP). These studies produced different results, even when using similar techniques, in attempting to demonstrate concordance between parasite genotypes and phenotypic expressions during infection. $T$. vaginalis genome composition provides a potential explanation for this difficulty in correlating genotype with phenotype. Trichomonas spp. genome is highly repetitive with $65 \%$ of the genome consisting of repeated sequences, including 59 repeat families of transposable elements representing $25 \%$ of genome. Although RAPD technique is recommended in phylogeny, OPD3 and OPD8 primers gave different reproducibility percentages amongst several similar previous studies (Vanacova et al, 1997; Kaul et al, 2004; Valadkhani et al, 2011), as well the present study. Similar repetitive families are present in other human parasites with clonal population structures as Leishmania, Plasmodium, Giardia, and Trypanosoma. http://www. a jtmh.org/content/80/2/245.long-ref-34. The ability of repeat elements to increase in copy number and transpose throughout genome would naturally have an increased impact on larger DNA fragments. According to the high similarity of RAPD bands, the present results agreed with Stiles et al. (2009). The recent advent of proteomic and genomic techniques based on $T$. vaginalis sequence presents an opportunity to elucidate the genetic factors controlling clinical manifestations, association with other disease entities, and drug resistance. Similarly a recent proteomic study on the effect of Tritrichomonas muris on mouse intestine (Kashiwagi et 
$a l, 2009)$ confirmed that 10 different proteins were expressed in T. muris infected mice intestines compared to non-infected mice intestines. The identified proteins represented several functions mainly related to stress, immune response, metabolism and signal transduction. Of these proteins is the heat shock protein (HSP27) which is down regulated in infected intestinal epithelial cell an effect similar to that occurs in amoebiasis, which implicit that T. muris injures and induces inflammation in the epithelium of the host tissue. Moreover, other trichomonads such as $T$. vaginalis and $T$. foetus produce similar effects on infected genital tract epithelium. The resultant relationships shown presently in gel sheets of RAPD technique as well as dendrogram clusters can explain the similar pathogenic effects of trichomonad species on the molecular genetic basis.

Snipes et al. (2000) used four OPD primers (OPD1, OPD2, OPD3, OPD5) to study heterogeneity of $T$. vaginalis metronidazole resistance in symptomatic women, the study resulted in that certain RAPD patterns to be associated with metronidazole resistance. Kaul et al. (2004) used five random primers (OPD1-OPD5) to study the heterogeneity of $T$. vaginalis between symptomatic and asymptomatic women, found that isolates from symptomatic women tended to form two distinct clusters, with OPD3 giving the least number of bands while OPD4 gave the highest number.

In the present study, 6 random primers (OPD1, OPD2, OPD3, OPD8,
TV2, \& TV6) were used to study the genetic relatedness of $T$. muris to $T$. vaginalis and to $T$. gallinae. The resulted showed marked genetic similarity of $>93 \%$ for OPD 3 and $>85 \%$ similarity for OPD8 between T. muris isolates and other isolates. For OPD1, only four isolates (Tmm11, Tmm12, Tmm14, \& Tmm17) had $>90 \%$ similarity, however, the remaining thirty six isolates had $>37 \%$ similarity (gave more divergent results). This showed that OPD1 primer was not a reproducible primer for further studies on T. $m u$ ris isolates. Valadkhani et al. (2011) used the same RAPD random primers to study geographical $T$. vaginalis genetic diversity between symptomatic and asymptomatic women and did not find much genetic diversity between the tested isolates.

Fraga et al. (2002) optimized the RAPD technique for Trichomonas by designing ten random primers for $T$. vaginalis (TV1-TV10), and by using this method; an association was found between genetic variability of $T$. vaginalis and the clinical presentation of infection. By using optimization factors mentioned in former study, the present one resulted in moderate reproducibility giving amplification fragments ranged from 200base pair to 450 base pair. Valadakhani et al. (2011) using same factors, resulted in higher reproducibility giving range of fragments 200-4200bp with OPD8 gave the least band number while OPD3 gave the greatest band number. Lawson et al. (2011) used OPD3, OPD5, OPD7, and OPD8 primers to characterize a 
novel $T$. gallinae strain resulted in no heterogeneity amongst the strains infecting British passerines. With RAPD giving fragment range of 92-999 and the OPD3 gave $>95 \%$ similarity which was higher than that of OPD8 which gave $>85 \%$ similarity, a result which nearly simulates the present result with $>93 \%$ similarity for OPD3 and $>85 \%$ similarity for OPD8 in addition to $>77 \%$ similarity of $T$. gallinae $\&>57$ $\%$ similarity of $T$. vaginalis to $T$. muris.

From a theoretical point of view, results of multilocus studies (like RAPD or isoenzyme analysis) reflect the real phylogeny of a taxon better than the results of single gene studies like rRNA sequencing because the cladogram of a particular gene (gene tree) may dramatically differ from the cladogram of species (species tree). Also the results of many previous experimental studies showed that the PCR fingerprinting can provide the valuable information about the phylogeny of a variety of groups of organisms, ranging from bacteria and protozoa to animals and plants (Ebert et al, 2002; Fry, 2003; terHorst, 2011). In this interspecies phylogenetic analysis the species of the same genera clustered together. It suggests that RAPD traits contain information on the phylogeny of trichomonads, an ancient and highly divergent group of clonal or semiclonal organisms, with $T$. gallinae isolate strain giving 57\% similarity with OPD3 and OPD 8 and $77 \%$ overall the similarity amongst the RAPD band pattern. However, T. vaginalis isolate showed 53\% similarity with OPD1, OPD3, \& OPD8 and with $87 \%$ overall similarity among the RAPD band pattern.

In the present multi-locus RAPD study using different random primers, the identity of RAPD patterns of different clones cultured on the same culture was very limited, if any, polymorphism within T. muris in vitro cultures. The RAPD analysis may be useful in epidemiologic studies, as RAPD analysis may be used to trace the spread of infection by a particular strain or to distinguish between a source and multisource infections, similar results on genetic diversity agreed with Vanacova et al. (1997) showed high statistical support for the close relationship of $T$. muris with $T$. vaginalis and $T$. gallinae.

\section{Conclusion}

An interesting outcome finding was the tight association between Tritrichomonas muris and Trichomonas gallinae. Further studies on phylogenecity as fingerprinting and sequencing of trichomonads namely human $T$. vaginalis, T. hominis and T. tenax, are ongoing to clear the doubts around sensitivity and specificity using the new molecular diagnostic tools.

\section{References}

Barretto, MP, Zago, F, De Oliveria, PV, et al, 1957: Study of vaginal trichomoniasis. I. Diagnostic methods. Rev. Assoc. Med. Bras. 3, 3:231-43.

Ebert, DC, Haag, JH, Riek, M, Pajunen, VI, 2002: A selective advantage to immigrant genes in a Daphnia metapopulation. Science 295:485-8

Fraga, J, Rojas, L, Sariego, I, Sarría, CA, 2002: Optimization of random amplified polymorphic DNA techniqu- 
es for use in genetic studies of Trichomonas vaginalis isolates. Infect. Genet. Evol. 2:73-5.

Frey, CF, Muller, N, 2012: Tritrichomonas-systematics of an enigmatic genus. Mol. Cell. Probes 26, 3:132-6.

Fry, JD, 2003: Detecting ecological trade-offs using selection experiments. Ecology 84:1672-8.

Jamali R, Zareikar, B, Kazemi, A, et al, 2005: Random amplified polymorphic DNA of Trichomonas vaginalis isolates from Tabriz, Iran. J. Parasit. Dis. 30, 1:53-7.

Kaul, P, Gupta, I, Sehgal, R, Malla, N, 2004: Trichomonas vaginalis: random amplified polymorphic DNA analysis of isolates from symptomatic and asymptomatic women in India. Parasitol. Int. 53, 3:255-62.

Kashiwagi, A, Hajime, K, Hong, LU, et al, 2009: Effects of Tritrichomonas muris on mouse intestine: a proteomic Analysis. Exp. Anim. 58, 5:537-42.

Lawson, B, Andrew, AC, Chantrey, J, Hughes, A, John, ShK, et al, 2011: A clonal strain of Trichomonas gallinae is the etiologic agent of an emerging avian epidemic disease. Infect. Genet. Evol. doi:10.1016/j.meegid.

Lynch, M, Milligan, BG, 1994: Analysis of population genetic structure with RAPD markers. Mol. Ecol. 3:917.

Mostegl, MM, Richter, B, Nedorost, N, Maderner, A, Dinhopl, N, et al, 2012: Identification of a putatively novel trichomonad species in the intes tine of a common quail (Coturnix coturnix). Vet. Parasitol. 183, 3/4:369-72

Perez, T, Albornoz, J, Dominguez, A
1998: An evaluation of RAPD fragment reproducibility and nature. Mol. Ecol. 7:1347-57.

Schmidt, GD, Roberts, LS, 1996: Flagellate Protozoa, Foundations of Parasitology, $5^{\text {th }}$ Ed. Times Mirror Co.

Snipes, LJ, Gamard, PM, Narcisi, E M, et al, 2000: Molecular epidemiology of metronidazole resistance in a population of Trichomonas vaginalis clinical isolates. J. Clin. Microbiol. 38: 3004-9.

Stabler, RM, 1951: A survey of Colorado band-tailed pigeons for Trichomonas gallinae. J. Parasitol. 37:471-3.

Stiles, JK, Shar, PH, Xue, L, et al, 2009: Molecular typing of Trichomonas vaginalis isolates by HSP70 restriction fragment length polymorphism. Am. J. Med. Hyg. 62:441-5.

terHorst, CP, 2011: Experimental evolution of protozoan traits in response to interspecific competition. J. Evol. Biol. 24:36-46.

Torres-Machorro AL, Hernandez, R, Alderet JF, Lopez, I, 2009: Comparative analysis among the Trichomonas vaginalis, Trichomonas tenax and Tritrichomonas foetus $5 \mathrm{~S}$ ribosomal RNA genes. Curr. Genet. 55:199-210.

Valadkhani, Z, Kazemi, F, Hassan, N, et al, 2011: Gene diversity of Trichomonas vaginalis isolates. Iran. J. Parasitol. 6, 3:101-6.

Vanacova, S, Tachezy, J, Kulda, J, Flegr, J, 1997: Characterization of trichomonad species and strains by PCR fingerprinting. J. Euk. Microbiol. 44: 545-52.

Wiley, EO, Lieberman BS, 2011: Phylogenetics: Theory and Practice of Phylogenetic Systematics. $2^{\text {nd }}$ ed. 